IP Periodica Polytechnica

Mechanical Engineering

61(2), pp. 122-129, 2017

https://doi.org/10.3311/PPme.9548

Creative Commons Attribution (i)

RESEARCH ARTICLE

\section{Establishment of a Beamforming Dataset on Basic Models of Low- Speed Axial Fan Blade Sections}

\author{
Esztella Balla $^{1 *}$, János Vad ${ }^{1}$
}

Received 03 June 2016; accepted after revision 09 January 2017

\begin{abstract}
The paper presents wind tunnel experiments, supplemented with phased array microphone measurements, on 2D basic models of low-speed axial fan blade sections: a flat plate, a cambered plate, and a RAF6-E airfoil. It aims at documenting the establishment of an acoustic beamforming dataset for the three profiles. The phased array microphone measurements offer spatially resolved information on the generated noise. The measurement setup enables the correlation of the streamwise evolution of the blade boundary layer with the associated noise characteristics. The dataset incorporates a wide range of incidence and Reynolds-numbers investigated. The present paper is confined to reporting on experimental results for arbitrarily selected representative incidences, Reynolds numbers, frequency bands, and profiles. The paper outlines a methodology for the evaluation and representation of the beamforming data in the following forms: source strength level based thirdoctave spectra obtained using background noise subtraction; maps presenting the loci of source strength level maxima; noise source maps for frequency bands of anticipated vortex shedding noise.
\end{abstract}

\section{Keywords}

beamforming, phased array microphones, fan noise, airfoil

\section{Introduction and objectives}

Low-speed axial flow fans are widely used in human environment. Some examples are as follows: fans in heating, ventilating and air conditioning (HVAC) for residential buildings as well as for non-residential buildings with occasional human access, and fans of industrial air technology (e.g. air supply, ventilation, cooling) operating in the vicinity of human personnel. In order to moderate the environmental impact of emitted fan noise on humans, and to ensure a proper aerodynamic operation as well, a concerted aeroacoustic and aerodynamic investigation and improvement of low-speed axial fans is a timely issue in the turbomachinery community.

In order to correspond to the simultaneous user demands of limitation in space and in rotor speed - being a basis for noise reduction as well -, the elemental blade sections of the aforementioned fans are often characterized by moderate Reynolds numbers, even below the critical value of $1.5 \cdot 10^{5}$ [1]. For achieving the prescribed aerodynamic performance - flow rate, total pressure rise - even at moderate diameter, rotor speed, and rotor blade count, such fans are often of high design specific performance, i.e. the blade sections are designed for high load (high lift). This corresponds to pronounced streamwise adverse pressure gradient on the blade suction side downstream of the suction peak. Such adverse effect is amplified due to the fact that the fan frequently operates in a throttled state relative to the design point, i.e. the flow incidence to the blade sections is increased. The resultant thickening or even separation of the suction side boundary layer tends to increase the turbulentboundary-layer-trailing edge noise and the separation-stall noise [1-2]. The aeroacoustic investigation of highly loaded / high incidence blade sections is therefore a topic of great practical importance, with special regard to discovering the correlation between the streamwise evolution of the boundary layer thickness and the spatial distribution of the noise sources associated with the thickened / separated boundary layer. In [3-5], a correlation has been found between the loss generated in the suction side boundary layer - represented by the momentum thickness - and the radiated broadband noise. This implies that by the detailed investigation of the boundary layer, additional 
knowledge can be gained regarding the noise formation mechanisms, contributing to the continuation of the research documented in [3-5]. Beyond the practical relevance as outlined above, the investigation of blade boundary layers exposed to pronounced adverse streamwise gradients, potentially leading to boundary layer separation, pose a challenge from basic research point view, in terms of experimentation, Computational Fluid Dynamics (CFD) as well as Computational Aeroacoustics (CAA) simulation.

Simple, traditional blade section geometry is characteristic for a great variety of axial fans, enabling relatively easy and low-cost manufacturing. Blades manufactured from e.g. sheet metal are characterized by cambered plate sections. Traditional airfoil profiles of relatively simple geometry - e.g. the RAF6$E$ profile, with a plain pressure surface [6] - are also popular in low-speed fan applications. It is important to note that for moderate Reynolds numbers, a cambered plate blade section may exhibit a more favorable aerodynamic behavior - higher possible lift, higher lift-to-drag ratio - than a profiled airfoil blade section. Examples are given for Reynolds numbers below $\approx 7 \cdot 10^{4}$ in [1], on the basis of [7]. The aforementioned favorable trends make the application of cambered plate blade sections especially relevant for certain low-speed fans, such as in [8].

In basic aerodynamic modelling of fan operation, the twodimensional (2D) airfoil / cascade approach is widely accepted [1]. In addition, also the basic modelling of the aeroacoustic behavior frequently relies on a $2 \mathrm{D}$ basis even in recent research projects. For example, in reference [9], the 2D blade elements are modelled acoustically as flat plates. Even recent experiments [10] aim at investigating the detailed aerodynamic features of the flat plate, however, without delivering spatially resolved measurement data on the acoustic behavior.

The above suggest the need for noise source localization for 2D basic models of blade sections, thus extending the benchmark database available in the literature for acoustic modelling of low-speed axial fans. The usage of phased array microphones (PAM) along with the beamforming technique is increasing in the field of noise source localization. Its advantage compared to measurements with individual microphones is that it offers a convenient way to spatially discretize the sound field. This recently emerging - and nowadays still relatively costly - technique, as such, has a number of applications e.g. in aerospace engineering, as e.g. in [11]. Only a few applications of PAM is reported in relationship with low-speed air-handling turbomachinery [12-15].

References [16-19] report on studies related to increased Reynolds numbers, i.e. being characteristic for the aeronautic industry, and related to moderate angles of attack. In [16-19], the PAM measurement planes were aligned either with the suction surface or with the pressure surface of the airfoils or fan blades. This configuration however does not provide acoustic information in correlation with the boundary layer as it evolves in chordwise and chord-normal direction, characterized e.g. by the boundary layer thickness.

In the beamforming measurement technique the evaluation of the measurement data is of great significance. Several deconvolution methods exist and the diagonal treatment of the cross spectral matrix can also be chosen. The diagonal removal was commonly used in recent studies [16-17, 19], however reference [20] challenges this practice by presenting its drawbacks.

To the authors' best knowledge, the PAM-based beamforming dataset established by the authors, and outlined in the paper, adds to the open literature from the viewpoint of the following objectives.

- It has been aimed to establish a concerted aeroacousticaerodynamic benchmark database on 2D basic models of low-speed axial fan blade sections, with involvement of the PAM technique for obtaining spatially resolved information on noise. The applicability of the PAM technique to this field of application - its advantages, and limits has been intended to be tested.

- The wind tunnel measurement setup presented in the paper enables the PAM investigation of the planes normal to the spanwise direction, thus correlating the chordwiseresolved and chord-normal resolved aeroacoustic and aerodynamic features.

- In the beamforming technique, instead of the diagonal removal method, the capabilities of another diagonal treatment method have been intended to be tested. More specifically, the original diagonal is retained, and optimized.

- The subjects of PAM investigation are basic models of low-speed axial fan blade sections, represented by three profiles: a flat plate, a cambered plate of circular arc camber line, and a RAF6-E profile. The three profiles are of rectilinear geometry, being in correspondence with the 2D flow approximation applied herein. To the authors' best knowledge, none of these geometries have been examined with the PAM technique; although aerodynamic and individual microphone measurement data are accessible for them, e.g. [21].

- The investigated Reynolds number range significantly extends below the critical value of $1.5 \cdot 10^{5}$.

- The range of studied angles of attack significantly exceeds the range presented in the literature [16-19]. Regarding the full dataset, the range of angle of attack $\alpha$ is between $-10^{\circ}$ and $50^{\circ}$, which means that extreme values are included as well - although only the range from $0^{\circ}$ to $30^{\circ}$ has been evaluated so far in detail. This examination range offers the possibility to take into account the effect of the angle of attack and to model a wide range of working conditions for fans, also including deep-stalled conditions. 


\section{Case studies}

The flat plate was investigated because, as it is the most basic geometry, it can serve as a reference in basic research (e.g. [9]), even though it is not used directly in fan aerodynamic design. However the cambered plate and the RAF6-E profile are still used in classic axial fan design, taking the advantage of being relatively easy to manufacture. The stacking lines of the studied profiles are perpendicular to the endwalls enclosing the profiles. The details of the profile geometries are presented in Table 1, where $c$ is the chord length, $s$ is the span, $t$ is the profile thickness, $h / c$ is the relative curvature of the camber line and LE and TE stands for leading and trailing edge, respectively. The cross-sections are shown in Fig. 1.

Table 1 The geometry and the material of the profiles

\begin{tabular}{lllllll}
\hline Profile & $c[\mathrm{~mm}]$ & $s[\mathrm{~mm}]$ & $t[\mathrm{~mm}]$ & $h / c[1]$ & LE, TE & Material \\
\hline Flat plate & 100 & 150 & 2.5 & 0 & sharp & aluminum \\
$\begin{array}{l}\text { Cambered } \\
\text { plate }\end{array}$ & 100 & 150 & 2.5 & 0.08 & sharp & aluminum \\
RAF6-E & 100 & 150 & $10 *$ & $0 * *$ & rounded & polymer \\
\hline
\end{tabular}

* Maximum thickness

** Uncambered; flat pressure surface

As suggested by [22], an aspect ratio $(A R)$ of at least 3 is beneficial for minimizing the three-dimensional flow (3D) effects. The $A R$ is only 1.5 for the experiments reported herein. This is an unavoidable compromise in the presented primary studies, constrained by the spanwise extension of the wind tunnel test section, the maximum available $U$, and the maximum targeted $\operatorname{Re}$ (tailoring $c$ ). [23] publishes $\alpha$-dependent $C_{\mathrm{L}}$ lift coefficient and $C_{\mathrm{D}}$ drag coefficient data for flat and cambered plates, for $R e=1.4 \times 10^{5}$. [23] reports on the influence of $A R$ on the lift and drag coefficients. These measurements show the following trends. a) For $\alpha \approx 0^{\circ}$ and $\alpha \approx 20^{\circ}$, the $3 \mathrm{D}$ lift and drag coefficients are in fair agreement with the $2 \mathrm{D}$ (i.e. infinite $A R$ ) data, for both $A R=3$ and $A R=1.5$. b) For the intermediate range of $\alpha \approx 10^{\circ}$, decreasing $A R$ from 3 to 1.5 increases the discrepancy between the $2 \mathrm{D}$ and $3 \mathrm{D} C_{\mathrm{L}}$ data only by about $25 \%$ as an average, and even much less increase of discrepancy can be observed between the 2D and $3 \mathrm{D} C_{\mathrm{D}}$ data. On this basis, despite the moderate $A R$, a 2D approach is considered reasonable in the present studies.

The profiles are equipped with cylindrical supporting struts on one side, which can be fitted into the bore on one of the walls of the test section. On the other side of the wall the angular position of the strut can be fixed by means of screws. The fixture allows the rotation of the profiles, and also contains a built-in protractor with which the desired angle of attack $\alpha$ can be set.

Based on e.g. [22], it is known that the use of a wind tunnel setup with a rectilinear blade section model involves a mean flow deviation due to the lateral momentum injection, corresponding to the lift force developing on the model. As a result, the effective inflow direction at a given angular position of the model differs from the idealistic inflow direction, represented by the longitudinal axis of the wind tunnel test section. Such flow deviation is to be taken into account when comparing the results to detailed simulations of the flow [22]. The aim of the illustrative case study presented herein is confined to qualitatively comparing the various profiles. For this reason, the aforementioned flow deviation was not taken herein into particular consideration. $\alpha$ has been defined as the geometrical angle between the chord line and the longitudinal axis of the test section.

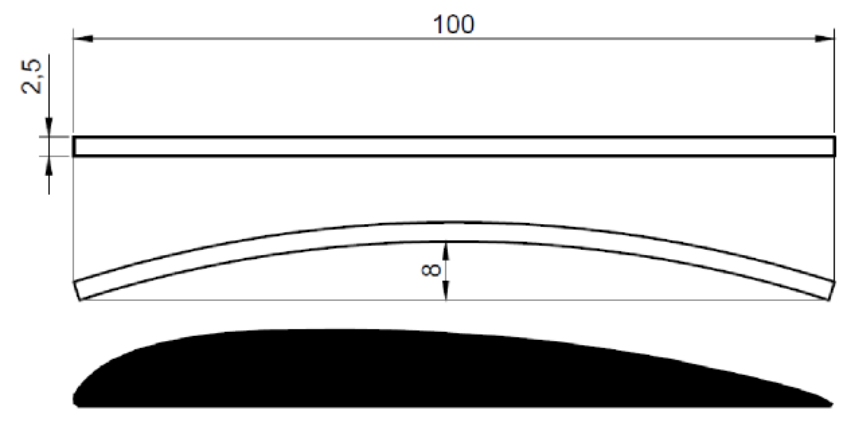

Fig. 1 The cross-sections of the flat plate (top), the cambered plate (middle) and the RAF6-E [6] (bottom)

The measurements were carried out at the flow conditions presented in Table 2. The $R e_{\text {min }}$ value is a representative value for small-scale fans, and is in approximate accordance with the $R e$ values valid for the experiments reported in [1] for a cambered plate profile. The $R e_{\max }$ value has been tailored by the available maximum air velocity in the wind tunnel. It is anyway in approximate correspondence with the critical value of $1.5 \cdot 10^{5}$, in [1], in the view of the note in [1] that even lower critical Reynolds number values are also appropriate in design of highly loaded blades. The $\alpha$ values are representing a wide range of operating conditions: the zero angle of attack can be considered a reference, while other angles are set for approximating the best lift-to-drag ratios of the profiles, and the highest angles represent the state of deep stall. The flow can be considered as incompressible, in accordance with the low Mach numbers.

Table 2 Flow conditions

\begin{tabular}{lllllll}
\hline \multicolumn{3}{c}{$\mathrm{Re}$} & \multicolumn{4}{c}{$\alpha$} \\
\cline { 1 - 5 } $\min$ & $\operatorname{mid}$ & $\max$ & $\min$ & $\operatorname{mid}$ & $\max$ & \\
\hline $6 \cdot 10^{4}$ & $10^{5}$ & $1.4 \cdot 10^{5}$ & $0^{\circ}$ & $10^{\circ}$ & $30^{\circ}$ & 0.06 \\
\hline
\end{tabular}

\section{Experimental setup}

The measurements were carried out in the Blackbird2 horizontal, blower-type wind tunnel of the Department [24]. The test section of the wind tunnel was closed only from one side. Opposite to the closed side a Kevlar wall was mounted. The noise of the flow field was measured by the microphone array 
through the Kevlar wall. The profiles were placed to the middle of the test section, and were bounded by the wall of the test section from one side and by a Kevlar wall on the other side. The distance between the midspan of the profiles and the microphone array was $0.556 \mathrm{~m}$. The test section of the wind tunnel ends at $4.5 c$ downstream of the TE of the profiles. The sketch of the measurement setup is shown in Fig. 2.

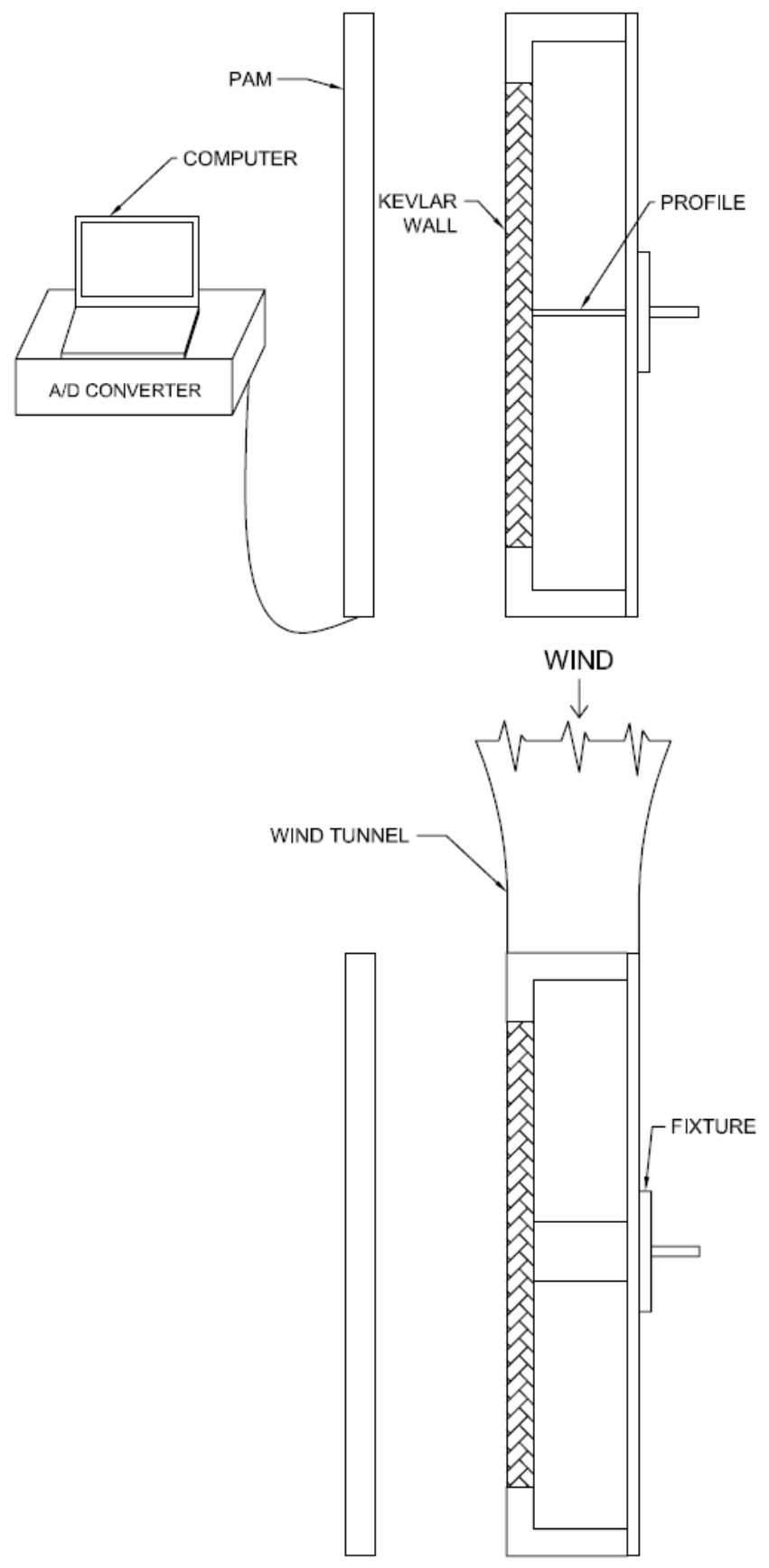

Fig. 2 Measurement setup from two perspectives

The Kevlar wall was placed between the test section and the microphone array as it imitates the aerodynamically impermeable side wall of the wind tunnel test section - being anyway a Plexiglass wall in routine applications of the wind tunnel, for optical access -, whereas it provides high acoustic transmission for the PAM experimentation. The Kevlar fabric was stretched on an aluminum frame evenly in all directions.

The turbulence intensity is $0.8 \%$ and the flow inhomogeneity is below $3 \%$ in the middle of the test section, with respect to the mean velocity in the test section. The turbulence intensity was calculated from one velocity component as the root-meansquare of the velocity fluctuation divided by the mean velocity. The properties of the flow field are presented in reference [24].

The uncertainty of setting the angle of attack $\alpha$ has been estimated to $\pm 0.5^{\circ}$. The inlet velocity in the test section, $v$, has been set with a conservatively estimated uncertainty of \pm $1.0 \%$, as checked with use of a calibrated Pitot-static probe.

An OptiNav Inc. Array24 general purpose PAM was used for noise recording. It consists of 24 microphones, arranged along a logarithmic spiral. The diameter of the PAM's aperture is 0.95 $\mathrm{m}$. A camera is situated in the center of the array, which takes pictures of the sound source while the microphones are recording the sound data. As the Kevlar wall blocks the view of the camera, the pictures had to be taken separately from the noise measurements without the Kevlar wall. The analogous signals of the microphones were amplified and converted to digital signals, which were then analyzed with a computer software. The imageJ image processing program with Beamform Interactive plug-in of OptiNav was used for the evaluation of the measurements. As reported in [5], the amplitude uncertainty of the source strength obtained by the PAM experiments has been estimated to $\pm 1 \mathrm{~d} B$. The duration of the measurements were $14 \mathrm{~s}$, with a sampling frequency of $44100 \mathrm{~Hz}$. Frequency domain beamforming was performed on the microphone data. The diagonal of the cross spectral density matrix was optimized. Beamforming was applied on a 2D grid at the midspan of the profiles.

The effect of the Kevlar wall was first investigated by measuring its sound attenuation at various frequencies. For this purpose the profiles were replaced with a point-like sound source which could emit sound at discrete frequencies. The measurement was made while the wind tunnel was turned off. The attenuation levels in the function of sound frequency (indicated with the center frequencies of the third octave bands) are shown in Fig. 3. The sound attenuation was defined by the difference between the source strength level (SSL) maxima detected by the PAM for the two cases: with and without the Kevlar wall. SSL is the output of the beamforming process [25], written in level form. The minimum of the investigated frequency range is in accordance with [5], while the maximum was adjusted to the capabilities of human audition. The attenuation of the Kevlar wall is frequency dependent, however comparison of the sound levels on the same frequencies are still possible without any compensation for the various profiles and flow conditions. 


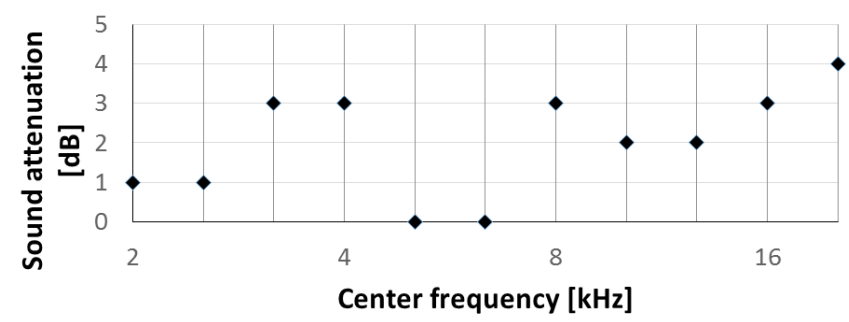

Fig. 3 Sound attenuation of the Kevlar wall in the function of frequency

\section{Methodology for data processing and representation}

The following figures serve as non-exhaustive illustrative examples for the steps of the methodology for data processing and representation. Measurements corresponding to $R e_{\text {mid }}$ are presented herein. Reference [26] gives an overview on the experiments related to $R e_{\max }$. The present paper is confined to discussing some arbitrarily selected specific results regarding the cambered plate and the RAF6-E airfoil, as these two profiles are representations for common low-speed axial fan blade geometries. A more comprehensive overview over the entire $R e$ and $\alpha$ range, involving all the three profiles, is a subject of future work.

Third octave spectra were created for both profiles and the background noise as well. The background noise includes the noise of the functioning wind tunnel, without a profile. The narrowband beamforming results serve as a basis for the spectra. Beamforming was applied to the whole frequency range in narrow $(21.5 \mathrm{~Hz})$ bands. The spectra are made up from the maxima belonging to each band. Beamforming was applied only to a confined region of the measurement plane. This region of interest (ROI) extends half $c$ upstream and one $c$ downstream of the LE and TE, respectively. It extends two $c$ in the vertical direction, with the profile being in the center.

Subtractive spectra were created, which are shown in Fig. 4. The subtractive spectra were obtained as follows. The logarithmic SSL values were converted to source strength values for the noise of the profiles and for the background noise. Then, the latter was subtracted from the former. The result was then converted again to a logarithmic level.

The following trends can be observed in the spectra. The explanation of the underlying physical phenomena requires a detailed, concerted aerodynamic and aeroacoustic analysis. As such, it forms the subject of future work.

At $\alpha=0^{\circ}$, in the lower frequency range, the noise level of the cambered plate is higher than of the RAF6-E. However this trend changes at approximately $2 \mathrm{kHz}$, and above this frequency the noise level of the RAF6-E is the higher.

At $\alpha=10^{\circ}$, at low frequencies, the noise levels for the RAF6-E are higher than those for the other two angles. A rapid decrease can be observed in the levels in point as the frequency increases. Above $2 \mathrm{kHz}$, the self-noise of the cambered plate becomes more significant.
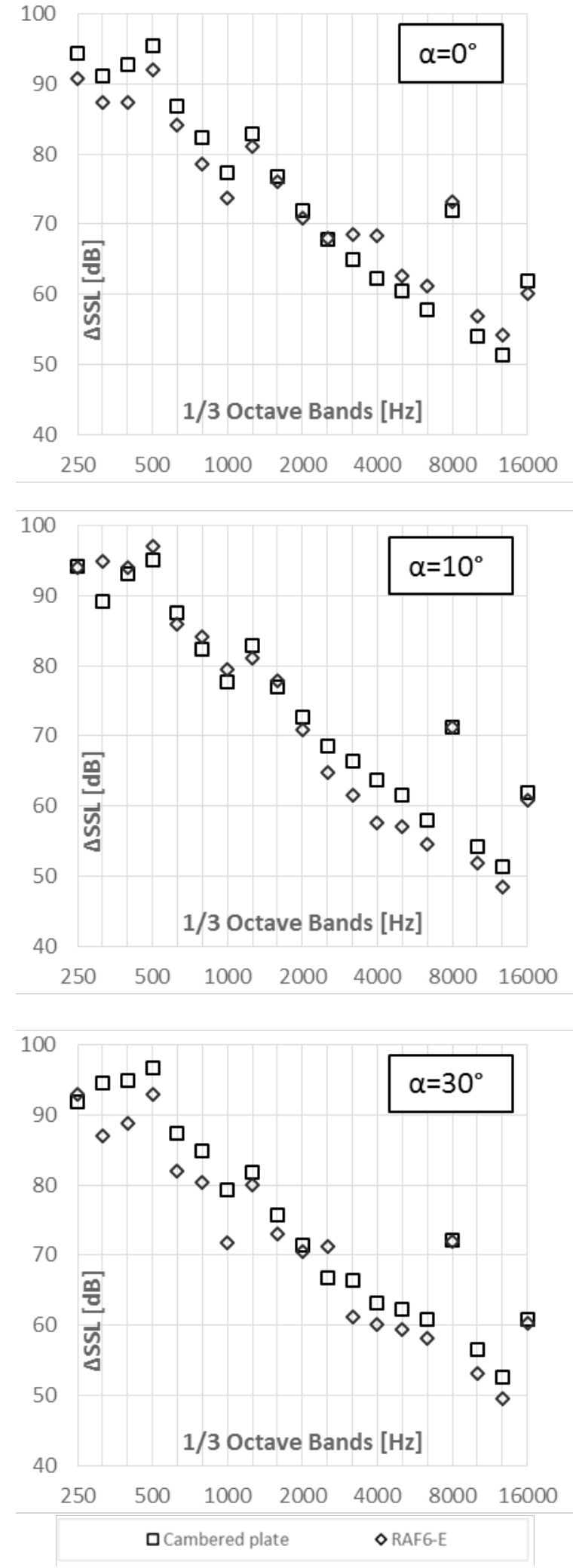

Fig. 4 Subtractive third octave spectra

At $\alpha=30^{\circ}$, throughout the entire presented frequency domain, the noise of the cambered plate is higher than of the RAF6-E (except for two bands).

In order to generally examine the location of broadband noise sources, maps of the loci of the narrowband beamforming maxima have been constructed, as presented in Fig. 5. The loci of maxima are indicated by crosses. Only those maxima are 
shown in Fig. 5 , which belong to frequencies above $5 \mathrm{kHz}$. This arbitrary choice is in accordance with the fact that the spectra in Fig. 4 suggest increased broadband noise in some bands above $5 \mathrm{kHz}$. A spatial constraint of peak representation was also applied arbitrarily as follows. For $\alpha=0^{\circ}$, maxima within the one-chord long region having the width of the profile thickness were represented. For $\alpha=10^{\circ}$ and $30^{\circ}$, the maxima within the + /- one profile thickness wide region of the bisector of $\alpha$ (relative to the horizontal direction) were represented. The profiles are indicated in the figure by their chord lines.

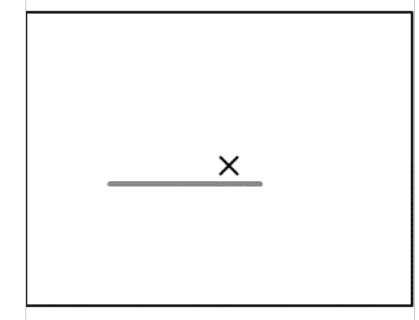

Cambered plate, $\alpha=0^{\circ}$

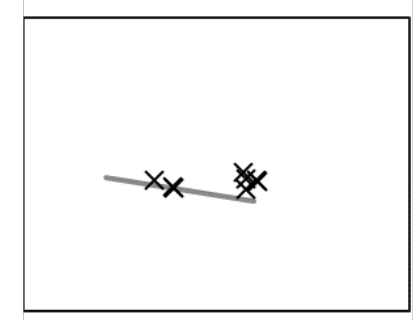

Cambered plate, $\alpha=10^{\circ}$

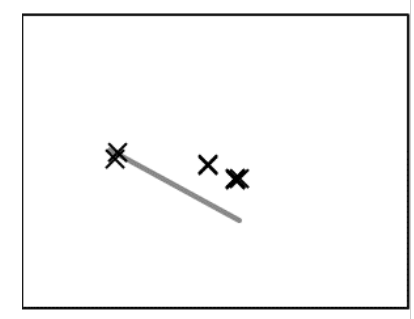

Cambered plate, $\alpha=30^{\circ}$

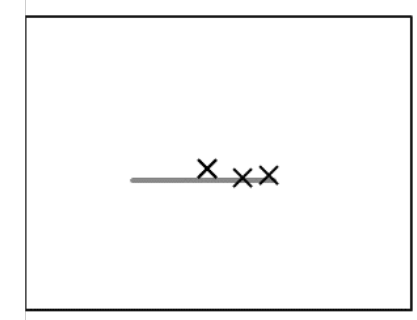

RAF6-E, $\alpha=0^{\circ}$

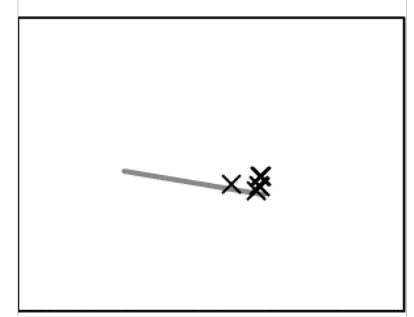

RAF6-E, $\alpha=10^{\circ}$

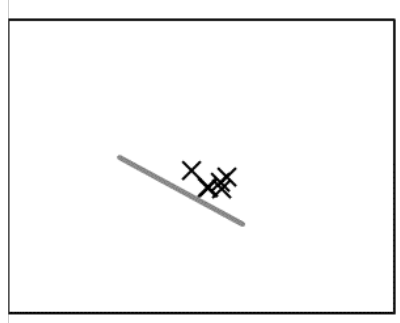

RAF6-E, $\alpha=30^{\circ}$
Fig. 5 Loci of representative narrowband maxima in the frequency range above $5 \mathrm{kHz}$

At $\alpha=0^{\circ}$ and $10^{\circ}$ the loci of the maxima for both profiles are dominantly close to the TE, which is presumably a sign of the TE-boundary layer interaction noise. In case of the cambered plate at $10^{\circ}$ angle of attack, maxima are also present closer to the LE. This may be associated with the laminar-turbulent transition of the boundary layer.

The separation noise seems to be the main noise source at $\alpha=30^{\circ}$ for both profiles. Noise sources near the LE also seem to be present in the case of the cambered plate.
$1345.8-1410.4 \mathrm{~Hz}$

$0.556 \mathrm{~m}$
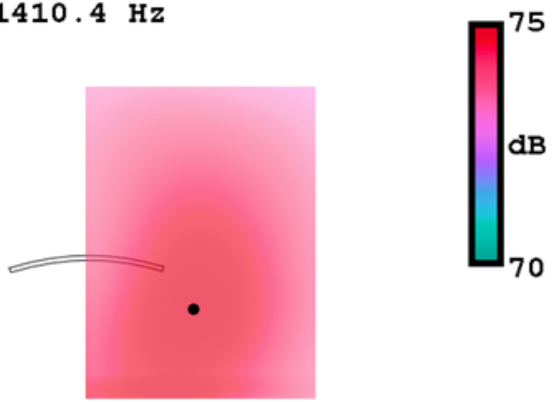

Cambered plate, $\alpha=0^{\circ}$

$3800.6-3865.2 \mathrm{~Hz}$

$0.556 \mathrm{~m}$
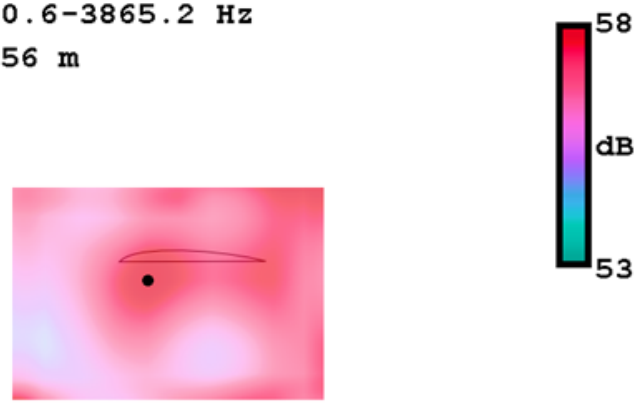

RAF6-E, $\alpha=0^{\circ}$

$1927.2-1991.8 \mathrm{~Hz}$

$0.556 \mathrm{~m}$
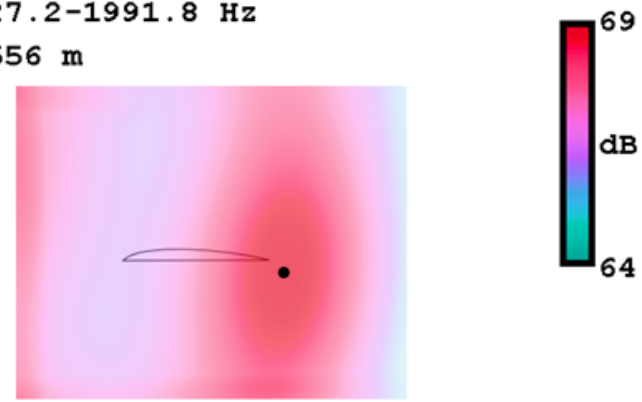

RAF6-E, $\alpha=0^{\circ}$

Fig. 6 Beamforming source maps: profile/blunt TE vortex shedding (top), blunt TE vortex shedding (middle), profile vortex shedding (bottom). The SSL maxima are marked with black dots.

Beamforming source maps were analyzed in order to find signatures of the vortex shedding noise, as a means for testing the source localization capabilities of the technique. Vortex shedding noise was chosen to be in the focus of our investigations, as the frequency at which it occurs can be easily determined by the corresponding Strouhal number $(S t) . S t$ is defined herein as $S t=f L / U . L$ is the characteristic length, which is dependent on the type of vortex shedding noise. Two types are distinguished: profile vortex shedding and blunt trailing edge vortex-shedding. In case of profile vortex shedding, $L$ is defined as the projection of the profile on the plane perpendicular to the mean flow [27]. For blunt trailing edge vortex-shedding, $L$ is the trailing edge thickness. By knowing or approximating $S t$, $f$ can be determined. According to [27], St can vary between 
0.8-1 for profile vortex shedding. Such approximation has been refined on the basis of the experiences gathered in evaluating the beamform results for $R e_{\text {max }}$, documented in [26]. For blunt trailing edge vortex-shedding, $S t$ is approximated as 0.2 [22]. With the approximate knowledge of $S t$, the third octave bands were identified within which vortex shedding is anticipated. At $\alpha=0^{\circ}$ both types of vortex shedding may occur. The estimated third octave band center frequencies for the cambered plate are $1.6 \mathrm{kHz}$ for both types of vortex shedding. For the RAF6-E the center frequencies are $2 \mathrm{kHz}$ and $4 \mathrm{kHz}$ respectively, for the profile vortex shedding and the for blunt trailing edge vortex-shedding. At $\alpha=10^{\circ}$ only the profile vortex shedding may occur, at a center frequency of $800 \mathrm{~Hz}$, however the resolution of the PAM technique at this low frequency, together with the high background noise, make it impossible to distinguish this noise source on the beamforming source maps.

The signatures of vortex shedding were sought in the aforementioned third octave bands. The results are shown in Fig. 6. In the frequency regions indicated on the maps visible signs of vortex shedding noise was found. An attempt has been made to increase the quality of the source maps by choosing a suitable ROI for each case. For the cambered plate (top graph), the two types of vortex shedding cannot be distinguished from each other on the basis of the source map, as their estimated frequencies are in the same third octave band. In case of the RAF6-E profile the two types could be separated. For the graph related to the blunt trailing edge vortex-shedding noise (middle graph), the SSL maximum is detected near the leading edge, and is associated with a source being different from vortex shedding. However, the local peak related to the vortex shedding noise is still visible near the trailing edge. The map regarding the profile vortex shedding noise of the RAF6-E airfoil (bottom graph) indicates the peak near the TE, in accordance with the assumption of occurrence of vortex shedding.

\section{Summary and future remarks}

This paper serves as a starting reference for the related future research. It aims at documenting the establishment of an acoustic beamforming dataset on representative 2D basic models of low-speed axial fan blade sections: a flat plate, a cambered plate, and a RAF6-E airfoil. The wind tunnel experiments, supplemented with a PAM measuring technique, are documented in detail.

To the authors' best knowledge, the established benchmark dataset adds to the open literature from the following perspectives: a) PAM-based, spatially resolved information on the noise generated by the aforementioned low-speed 2D blade sectional models. b) Offering the possibility for correlating the streamwise evolution of the blade boundary layer with the associated noise characteristics. c) Extension of the ranges of Reynolds number and flow incidence under investigation, being especially relevant for low-speed axial fan blades.

The methodology for data processing and representation, to be used in future evaluation of the results, is outlined herein. The methodology is illustrated by means of representative examples on the following: a) Comparative spectra based on the detected SSL maxima. b) Maps presenting the loci of the SSL maxima in narrowband beamforming. c) Noise source maps.

In future work, the following steps of acoustic data processing are envisaged, with regard to evaluating the results over the entire dataset. a) Elaboration and application of a Fourier analysis-based technique for enhancement of the remarkable noise sources within specific frequency bands, and for effective removal of perturbations (e.g. background noise, sidelobes). b) Fuzzy clustering for identification of dominant noise sources that extend spatially as well as in frequency. c) Quantification of the broadband noise sources of various origin, thus contributing to the extension and refinement of semi-empirical acoustic models available in the literature for low-speed axial fans.

\section{Nomenclature}

\section{Abbreviations}

AR

CAA

CFD

HVAC

LE

PAM

ROI

SSL

\section{TE}

\section{Latin letters}

$c$
$C_{L}$
$C_{D}$
$f$
$h$
$h / c$
$L$
$M a$
$R e$
$S t$
$s$
$t$
$U$
Greek letters
$\alpha$
$v$
Subscripts
max
mid
min

aspect ratio $=\mathrm{s} / \mathrm{c}$

computational aeroacoustics

computational fluid dynamics

heating, ventilating and air conditioning leading edge phased array microphone region of interest source strength level (level of beamforming based local source strength normalized by a reference value)

trailing edge

chord length [mm]

lift coefficient [1]

drag coefficient [1]

vortex shedding frequency $[\mathrm{Hz}]$

maximum height of the camber line [mm]

relative curvature of camber line [1]

characteristic length [m]

Mach number [1]

chord based Reynolds number $=\mathrm{cU} / \mathrm{v}[1]$

Strouhal number $=\mathrm{fL} / \mathrm{U}[1]$

span [mm]

profile thickness [mm]

inlet velocity in the test section $[\mathrm{m} / \mathrm{s}]$

angle of attack $\left[^{\circ}\right]$

kinematic viscosity $\left[\mathrm{m}^{2} / \mathrm{s}\right]$

maximum value of the range

intermediate value of the range

minimum value of the range 


\section{Acknowledgements}

This work has been supported by the Hungarian National Research, Development and Innovation Centre under contract No. K 112277.

The work relates to the scientific programs "Development of quality-oriented and harmonized $\mathrm{R}+\mathrm{D}+\mathrm{I}$ strategy and the functional model at BME" (Project ID: TÁMOP4.2.1/B-09/1/KMR-2010-0002) and "Talent care and cultivation in the scientific workshops of BME" (Project ID: TÁMOP4.2.2/B-10/1-2010-0009).

\section{References}

[1] Carolus, T. "Ventilatoren." Teubner Verlag. 2003. (in German)

[2] Brooks, T. F., Pope, D. S., Marcolini, M. A. "Airfoil self-noise and prediction." NASA Reference Publication NASA-RP-1219. 1989.

[3] Benedek, T., Vad, J. "Concerted aerodynamic and acoustic diagnostics of an axial flow industrial fan, involving the phased array microphone technique." In: ASME Turbo Expo 2014: Turbine Technical Conference and Exposition. Vol. 1A: Aircraft Engine; Fans and Blowers, Düsseldorf, Germany, June 16-20, 2014

https://doi.org/10.1115/GT2014-25916

[4] Benedek, T., Vad, J. "Spatially resolved acoustic and aerodynamic studies upstream and downstream of an industrial axial fan with involvement of the phased array microphone technique." In: ETC'11 - The 11th European Conference on Turbomachinery Fluid Dynamics and Thermodynamics, Madrid, Spain, 23-27 March 2015, Paper \# 128, 11p. 2015.

[5] Benedek, T., Vad, J. "An industrial on-site methodology for combined acoustic-aerodynamic diagnostics of axial fans, involving the Phased Array Microphone technique." International Journal of Aeroacoustics. 15(1-2), pp. 81-102. 2016.

https://doi.org/10.1177/1475472X16630849

[6] Vad, J., Koscsó, G., Gutermuth, M., Kasza, Zs., Tábi, T., Csörgő, T. "Study of the aero-acoustic and aerodynamic effects of soft coating upon airfoil." JSME International Journal Series C-Mechanical Systems Machine Elements and Manufacturing. 49(3), pp. 648-656. 2006. https://doi.org/10.1299/jsmec.49.648

[7] Albring, W. "Angewandte Strömungslehre." 6th edition. Akademie-Verlag Berlin. 1990

[8] Vad, J., Horváth Cs., Kovács J. G. "Aerodynamic and aero-acoustic improvement of electric motor cooling equipment." Journal of Power and Energy. 228(3), pp. 300-316. 2014. https://doi.org/10.1177/0957650913517678

[9] Sturm, M., Sanjosé, M., Moreau, S., Carolus, T. "Application of Analytical Noise Models Using Numerical and Experimental Fan Data." In: ETC'11 - The 11th European Conference on Turbomachinery Fluid Dynamics and Thermodynamics, Madrid, Spain, March 23-27, 2015, Paper \# 241, 13p. 2015

[10] Jones, A. R., Medina, A., Spooner, H., Mulleners, K. "Characterizing a burst leading-edge vortex on a rotating flat plate wing." Experiments in Fluids. 57(4). 2016. https://doi.org/10.1007/s00348-016-2143-7

[11] Horváth, Cs. "Beamforming Investigation of Dominant Counter-Rotating Open Rotor Tonal and Broadband Noise Sources." AIAA Journal. 53(6), pp. 1602-1611. 2015. https://doi.org/10.2514/1.J053465

[12] Zenger, F., Junger, C., Kaltenbacher, M., Becker, S. "A Benchmark Case for Aerodynamics and Aeroacoustics of a Low Pressure Axial Fan." SAE Technical Paper, No. 2016-01-1805. 2016. https://doi.org/10.4271/2016-01-1805
[13] Migliore, P., Oerlemans, S. "Wind tunnel aeroacoustic tests of six airfoils for use on small wind turbines." Journal of Solar Energy Engineering. 126(4), pp. 974-985. 2004.

[14] Moreau, D. J., Prime, Z., Porteous, R., Doolan, C. J., Valeau, V. "Flow-induced noise of a wall-mounted finite airfoil at low-to-moderate Reynolds number." Journal of Sound and Vibration. 333(25), pp. 6924-6941. 2014. https://doi.org/10.1016/j.jsv.2014.08.005

[15] Minck, O., Binder, N., Cherrier, O., Lamotte, L., Budinger, V. "Fan Noise Analysis Using a Microphone Array." In: Proceedings of the International Conference on Fan Noise, Technology and Numerical Methods (FAN2012), Senlis, France, 2012.

[16] Hutcheson, F. V., Brook, T. F. "Effects of angle of attack and velocity on trailing edge noise determined using microphone array measurements." International Journal of Aeroacoustics. 5(1), pp. 39-66. 2006. https://doi.org/10.1260/147547206775220425

[17] Geyer, T., Sarradj, E., Giesler, J. "Application of a Beamforming Technique to the Measurement of Airfoil Leading Edge Noise." Advances in Acoustics and Vibration. Vol. 2012, Article ID 905461, 2012. https://doi.org/10.1155/2012/905461

[18] Padois, T., Laffay, P., Idier, A., Moreau, S. "Detailed experimental investigation of the aeroacoustic field around a Controlled-Diffusion airfoil." In: 21st AIAA/CEAS Aeroacoustics Conference, AIAA AVIATION Forum, AIAA Paper 2015-2205. Dallas, TX, 2015. https://doi.org/10.2514/6.2015-2205

[19] Moreau, D. J., Doolan, C. J. "Tonal noise production from a wall-mounted finite airfoil." Journal of Sound and Vibration. 363, pp. 199-224. 2016. https://doi.org/10.1016/j.jsv.2015.11.021

[20] Dougherty, R. P. "Cross spectral matrix diagonal optimization." BeBec Paper 2016-S2. 2016.

[21] Spedding, G. R., McArthur, J., Rosén, M. "Deducing aerodynamic mechanisms from near- and farwake measurements of fixed and flapping wings at moderate Reynolds number." In: 44th AIAA Aerospace Sciences Meeting and Exhibit, Aerospace Sciences Meetings, AIAA Paper 2006-33. Reno, Nevada, USA, Jan. 9-12, 2006. https://doi.org/10.2514/6.2006-33

[22] Roger, M., Moreau, S. "Extensions and limitations of analytical airfoil broadband noise models." International Journal of Aeroacoustics. 9(3), pp. 273-305. 2010. https://doi.org/10.1260/1475-472X.9.3.273

[23] Mueller, T. J. "Aerodynamic measurements at low Reynolds numbers for fixed wing microair vehicles." In: RTO AVT/VKI Special Course, Development and operation of UAVs for military and civil applications, VKI, Belgium, Sept. 13-17, 1999.

[24] Gulyás, A., Balczó, M. "Development of a Small Blower-type Wind Tunnel for Educational Purposes." In: 28th microCAD International Multidisciplinary Scientific Conference, 2014.

[25] Dougherty, R. P. "Beamforming in Acoustic Testing." In: Experimental Acoustics. (Mueller, T. J. (ed.)) Springer, Berlin, 2002, pp. 62-97. https://doi.org/10.1007/978-3-662-05058-3

[26] Balla, E., Vad, J. "Beamforming studies on basic models of low-speed axial fan blade sections." In: ETC'12 - The 12th European Conference on Turbomachinery Fluid Dynamics and Thermodynamics, Stockholm, Sweden, April 3-7, 2017, Paper \# 119, 13p., 2017.

[27] Yarusevych, S., Boutilier, M. S. H. "Vortex shedding characteristics of a NACA 0018 Airfoil at low Reynolds numbers." In: 40th Fluid Dynamics Conference and Exhibit, Fluid Dynamics and Co-located Conferences, AIAA Paper 2010-4628, Chicago, Illinois, 28 June - 1 July 2010. https://doi.org/10.2514/6.2010-4628 